\title{
Gold Nanosensitisers for Multimodal Optical Diagnostic Imaging and Therapy of Cancer
}

Vijay Raghavan ${ }^{1}$, Jennifer M Connolly ${ }^{1}$, Hai Ming Fan², Peter Dockery ${ }^{3}$, Antony Wheatley ${ }^{4}$, Ivan Keogh ${ }^{5}$ and Malini Olivo ${ }^{1,6,7, *}$

${ }^{1}$ School of Physics, National University of Ireland Galway, Galway, Ireland

${ }^{2}$ School of Chemical Engineering, Northwest University, Xi'an, P. R. China

${ }^{3}$ Anatomy, National University of Ireland, Galway

${ }^{4}$ Physiology, National University of Ireland, Galway

${ }^{5}$ Department of Surgery, National University of Ireland, Galway

${ }^{6}$ Royal College of Surgeons (RCSI), Dublin, Ireland

${ }^{7}$ Bio-optical Imaging Group, Singapore Bioimaging Consortium, Agency for Science, Technology and Research, Singapore

\begin{abstract}
Multi-modal nanoprobes have attracted much attention because of their superior chemical and physical properties that have great potential for early diagnosis and treatment of cancer. We report herein a novel stepwise fabrication of a gold nanoparticle-Raman reporter-photosensitiser conjugate called a Gold Nanosensitiser (NS). The efficacy of gold NS as multimodal nanoprobes for surface enhanced Raman spectroscopy (SERS) imaging and photodynamic (PDT) and photothermal treatment (PTT) of cancer were examined. NS were constructed using a multilayer technique in which a Raman reporter, DTTCI (3,3'-Diethylthiatricarbocyanine iodide) and a photosensitiser, hypericin, were coated onto gold nanostars. By adjusting the layer spacing, these coatings endowed NS with both enhanced SERS and fluorescence bioimaging capabilities. Excitation of the NS in solution at an appropriate wavelength permitted examination of the photodynamic therapy (PDT) capability in terms of singlet oxygen generation. As the nanostar is multibranched and comprises multiple 'hot spots' capable of enhanced electromagnetic radiation, along with SERS it also exhibits photothermal therapy (PTT) capability when excited at its resonant wavelength of $675 \mathrm{~nm}$. The NS was also characterised to examine the retention of physio-chemical and optical properties of its components after fabrication. The development of such a multimodal nanoplatform in the current work will offer a useful tool for noninvasive, high-accuracy, single-node diagnosis and therapy of cancer.
\end{abstract}

Keywords: Gold Nanostar; Photosensitiser; Hypericin; Multi-modal imaging; Photodynamic therapy; Photothermal therapy; SERS imaging

\section{Introduction}

In the recent past, there have been accelerated developments in biomedical modalities for the early diagnosis and therapy of cancer. Of primary interest in cancer research is the conservation of healthy tissue during therapeutic efforts, in addition to minimally- or noninvasive diagnosis. Photodynamic therapy (PDT) is one such treatment modality that takes advantage of a light absorbing class of chemicals called photosensitisers (PS) [1]. When excited with light at an appropriate wavelength, PS can generate cytotoxic singlet oxygen and other reactive oxygen species (ROS). Most of the PSs are hydrophobic in nature which makes systemic administration problematic and, in turn, affects its delivery to and uptake by tumour cells $[2,3]$. Thus, they tend to aggregate, which reduces the quantum efficiency of ROS generation. This has led to the development of third-generation PS that are now equipped with nanocarrier systems to enhance their targeted delivery for efficient PDT and photodynamic diagnosis (PDD). These nanocarriers, which load PS by physisorption or covalent binding, offer benefits of hydrophilicity and appropriate size for passive targeting of tumour by the enhanced permeability and retention (EPR) effect. Various types of nanocarriers are available and their use in PDT has been extensively reviewed [4]. In particular, gold nanoparticles are favoured as PS carrier due to their biocompatibility, easy preparation, efficient bioconjugation and high-drug loading capacity [4,5]. Also strong absorption and scattering of gold nanoparticles provides opportunities for contrast enhanced optical imaging.

Various anisotropic structures of gold nanoparticles with superior optical properties have been reported that includes nanowires, nanorods, nanocubes, nanoplates, etc [6,7]. Multibranched star-shaped nanoparticles bearing anisotropic 3D nanocrystals exhibit interesting optical properties that can be fine-tuned by adjusting the geometry in terms of aspect ratio of the branches. When in resonance with the incident light, nanostars exhibit intense surface plasmons at the tips of the branches called hot spots. This acts to significantly enhance the electromagnetic field intensity. This property called localised surface plasmon resonance (LSPR) in turn enhances the scattering crosssection of the nanostars and of the molecules adsorbed, an effect called surface enhanced Raman scattering (SERS) [8-10]. Bioimaging based on SERS tags has attracted growing interest recently. It has shown many advantages over other optical bioimaging techniques including the multiplexing capability due to the narrow width of Raman bands, better photostability of the Raman active molecules, higher spatial resolution due to the confinement of the intensified electric field around the surface of the nanoparticles, and high signal-to-noise ratio in complex biological systems at single wavelength excitation in the near infrared (NIR) region $[11,12]$

At SPR frequency, nanostars convert the strongly absorbed radiation into heat by electron-phonon and phonon-phonon processes [13]. Nanostars thus serve as heating antennas that lead to elevated temperature of the surrounding medium and induce hyperthermia or

${ }^{*}$ Corresponding author: Malini Olivo, School of Physics, National University of Ireland Galway, Galway, Ireland, Tel: 353-91-492515; E-mail: Malini_Olivo@sbic.a-star.edu.sg

Received August 28, 2014; Accepted October 15, 2014; Published October 24, 2014

Citation: Raghavan V, Connolly JM, Fan HM, Dockery P, Wheatley A, et al. (2014) Gold Nanosensitisers for Multimodal Optical Diagnostic Imaging and Therapy of Cancer. J Nanomed Nanotechnol 5: 238. doi: 10.4172/2157-7439.1000238

Copyright: @ 2014 Raghavan V, et al. This is an open-access article distributed under the terms of the Creative Commons Attribution License, which permits unrestricted use, distribution, and reproduction in any medium, provided the original author and source are credited. 


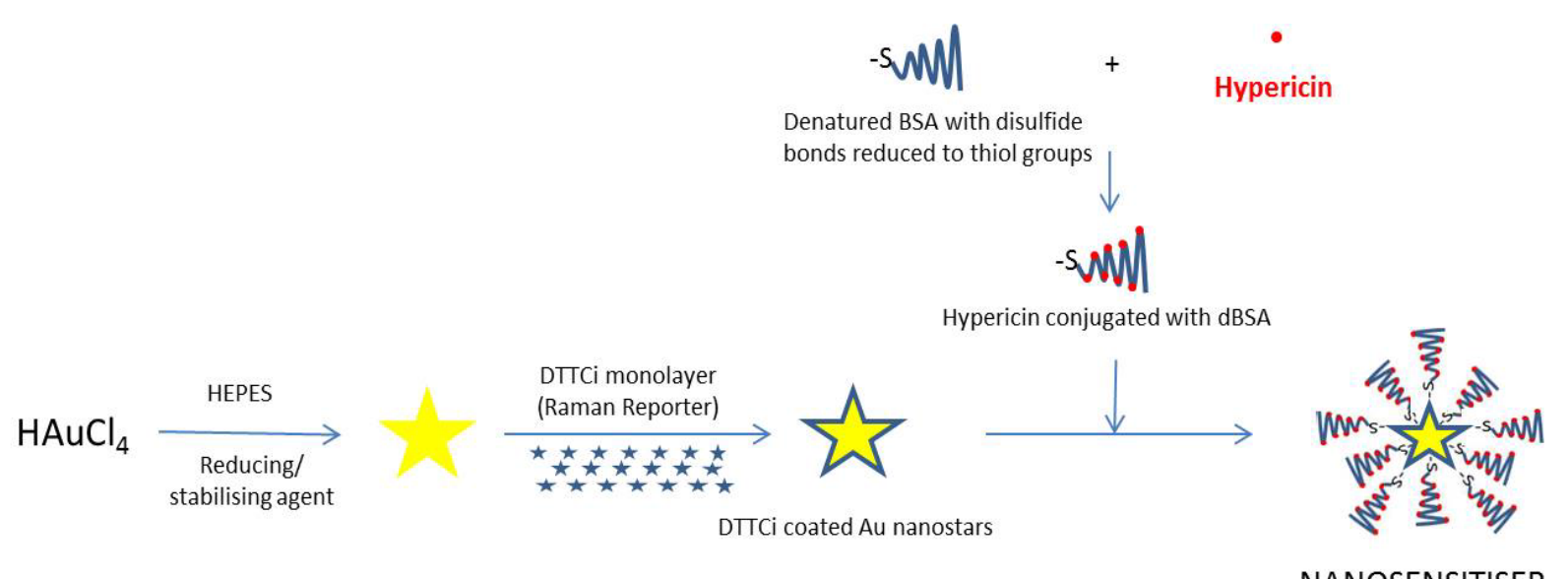

Figure 1: Schematic representation of fabrication of Nanosensitiser.

irreversible thermal damage to the targeted tissue, thereby offering an alternative cancer treatment called photothermal therapy (PTT). Recent studies reported that combined PDT and PTT treatment modalities to be an effective treatment strategy compared to conventional PDT or PTT alone [14-16].

Hypericin is a naturally occurring plant-based perylene-quinone dye that has been successfully tested as an effective photosensitiser in in-vitro studies [17-19] due to its properties of fast clearance, low dark toxicity and higher quantum efficiency of reactive oxygen species generation [20-23]. Herein, as a proof-of-concept, we propose fabrication of a nanoprobe, called a Nanosensitiser (NS). This NS comprises of a gold nanoparticle core that when coated with a Raman reporter and hypericin, enhances both the SERS signal of the reporter and the delivery of the photosensitiser. Such a NS has applications in multimodality optical imaging of cancer including SERS and fluorescence imaging, and therapy of cancer including PDT and PTT. The development of such a multimodal nanosensitiser in this current work will offer a useful tool for non-invasive, high-accuracy, singlenode detection and therapy of cancer.

\section{Materials and Methods}

All the chemicals used in this study were purchased from Sigma Aldrich and deionised water was used as solvent. All glassware used was washed with aquaregia (mixture of 3:1 ratio of hydrochloric and nitric acid, respectively) and rinsed with Millipore ultrapure water.

\section{Nanoparticle synthesis}

One-pot synthesis of 3-dimensional multibranched gold nanocrystals was utilised based on the green chemistry approach [24], where the common biochemical buffer, 4-(2-Hydroxyethyl)piperazine1-ethanesulfonic acid (HEPES) was used as a weak reducing and shape directing agent. An aqueous solution of HEPES $(40 \mathrm{mM} ; \mathrm{pH}$ 7.4) was mixed with aqueous tetrachloroauric acid $\left(\mathrm{HAuCl}_{4}\right)$ at room temperature so that the final concentration of $\mathrm{HAuCl}_{4}$ was $200 \mu \mathrm{M}$. The colour of the reaction solution turned greenish-blue within 30 minutes signalling the formation of branched nanocrystals. Reaction parameters including the concentration ratio of gold precursor to reducing agent $\left(\mathrm{R}_{[\mathrm{HEPES}] / \mathrm{HAuCl} 4]}\right), \mathrm{pH}$ and temperature were varied to obtain multibranched gold nanoparticles with desired surface plasmon absorption wavelength.

\section{Nanoparticle characterisation}

Absorption and fluorescence measurements were performed using the Shimadzu UV-2600 and RF-5301 spectrophotometers, respectively. The size and morphology of nanoparticles were examined using the Hitachi H7000 transmission electron microscope (TEM) with accelerating voltage of $75 \mathrm{kV}$. Surface enhanced Raman scattering (SERS) measurements were carried out with Renishaw Raman spectrometer with Invia microscope. The excitation source was a 785 $\mathrm{nm}$ diode laser with samples focused using 20x objective. The elemental gold concentration was quantified using Varian SpectrAA 200 atomic absorption spectrophotometer (AAS). Singlet oxygen generation by hypericin in the nanoprobe was examined at an excitation of $543 \mathrm{~nm}$ with a HeNe fibre optic laser coupled with a collimator. Temperature increase in solution by gold nanoparticles was measured with a digital temperature probe using a $675 \mathrm{~nm}$ laser excitation.

\section{Nanoprobe fabrication}

Nanoparticles were recovered from the reaction solution by centrifugation and re-dispersed in water. The Raman reporter, 3,3' diethylthiatricarbocyanine iodide (DTTCI), was then added dropwise to the colloidal nanoparticles under vigorous stirring. Bovine serum albumin (BSA) was chemically denatured by adding sodium borohydride $\left(\mathrm{NaBH}_{4}\right)$ in aqueous condition at $70-75^{\circ} \mathrm{C}$. Unreacted sodium borohydride was removed by spontaneous decomposition [25]. Hypericin was added to denatured BSA (dBSA) and left overnight for incubation [26]. The dBSA:Hyp complex was purified by removing unbound hypericin using Nanosep ${ }^{\text {Tx }} 10000$ MWCO OMEGA membrane (Pall Life Sciences). DTTCI tagged nanostars were then centrifuged and re-dispersed in dBSA:Hyp solution and left overnight at room temperature. Fabricated nanoprobes were characterised for physiochemical and optical properties. The schematic of nanoprobe fabrication can be seen in Figure 1.

\section{In-vitro studies}

A human oral cancer cell line (SCC9) was purchased from American Type Culture Collection (ATCC). Cells were cultured under standard conditions $\left(5 \% \mathrm{CO}_{2}, 95 \% \mathrm{O}_{2}, 37^{\circ} \mathrm{C}\right)$ in Dulbecco's modified Eagle's medium (DMEM)/nutrient mixture F-12 Hams, phenol free (Sigma, D6434) supplemented with foetal bovine serum (10\%; SigmaAldrich), L-glutamine (2.5 mM; Sigma-Aldrich), penicillin (100U/ 


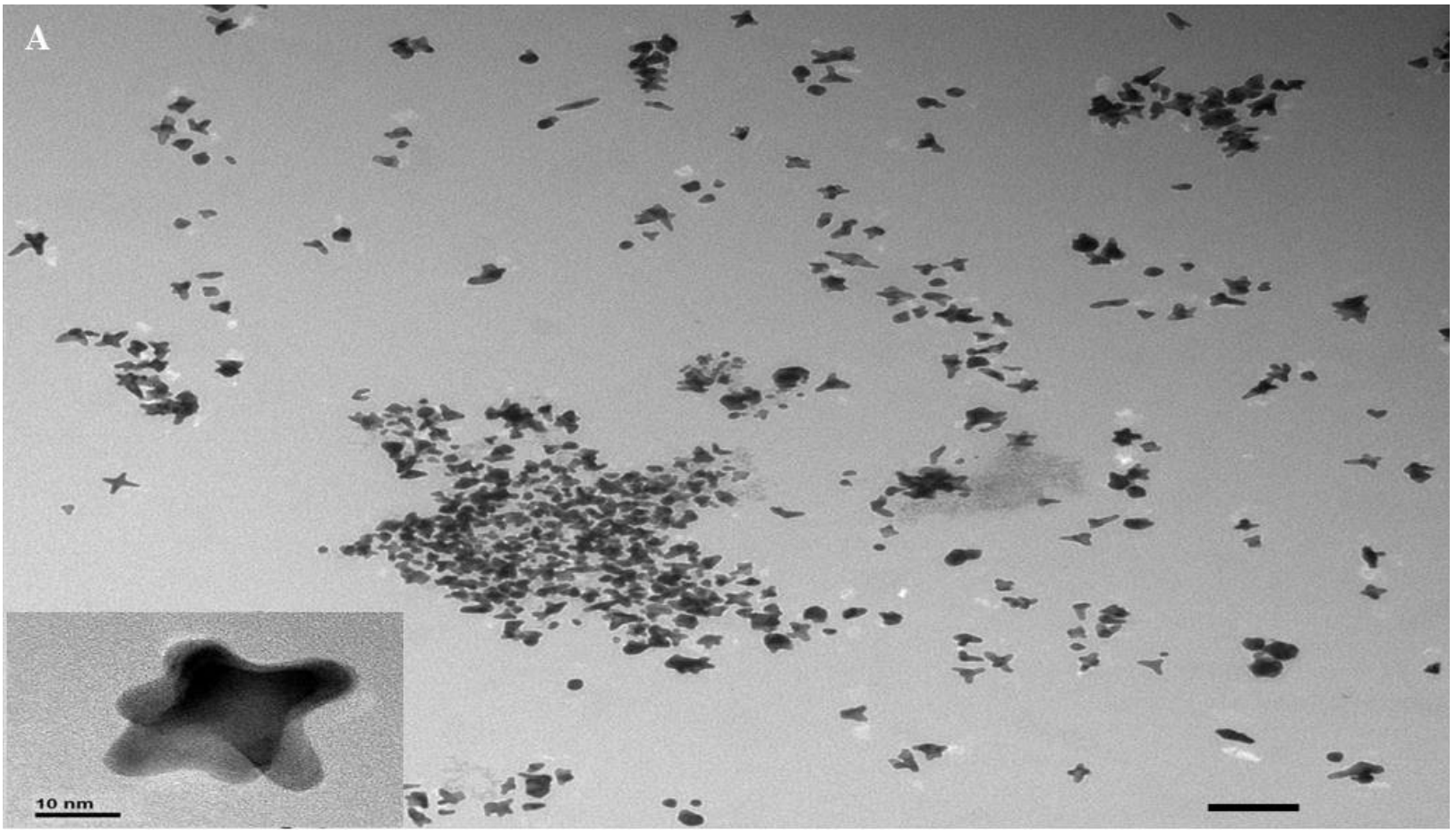

B

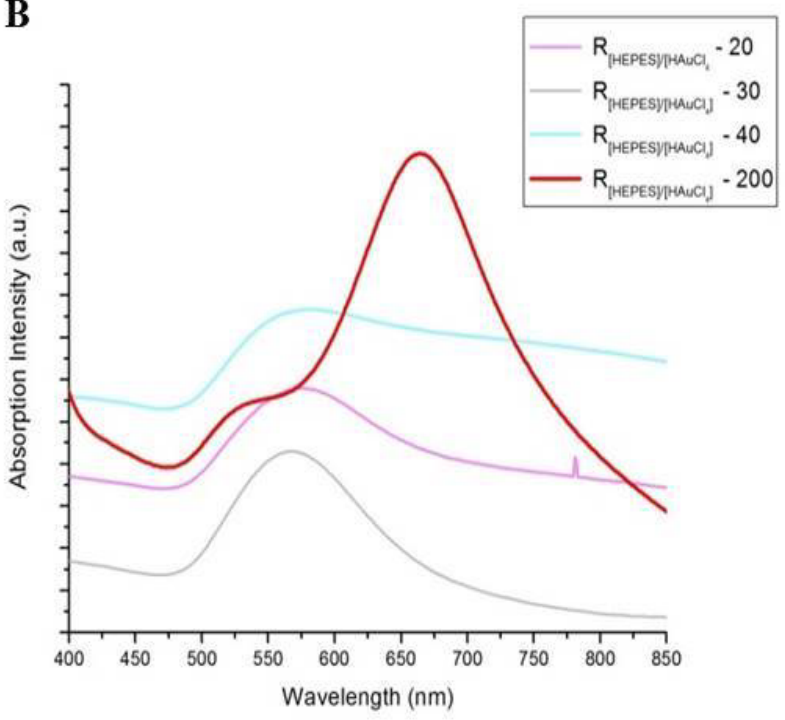

C

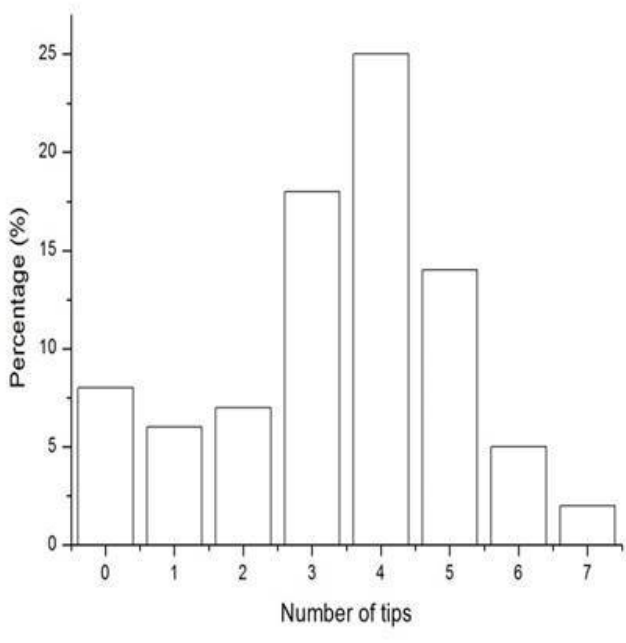

Figure 2: Characterisation of Gold nanostars - TEM image of gold nanostars synthesised with $R_{[H E P E S /[H A U C \mid 4]}$ of 200 (scale bar: $100 \mathrm{~nm}$ ). HRTEM image of a single gold nanostar inset (A), Absorption spectra of gold nanostars synthesised with different concentration ratio of gold precursor to reducing agent (B), and distribution of gold nanostars based on number of tips (C).

$\mathrm{ml}$; Sigma-Aldrich), streptomycin (100 $\mu \mathrm{g} / \mathrm{ml} ; \quad$ Sigma-Aldrich). Confocal fluorescence microscopy (CFM) was performed using Andor Revolution Confocal Microscope with motorised inverted microscope fitted with a piezo controlled z-stage. Darkfield microscopy (DFM) was carried out with a light microscope fitted with Cytoviva advanced darkfield illumination system that replaces the standard condenser. For both CFM and DFM, cells were cultured in 8-well plates and fixed with paraformaldehyde after incubation with NS. Fluoromount mounting medium was added before placing of a Quartz cover slip for imaging. For PDT and PTT studies, cells were seeded at a concentration of $5 \times 10^{3}$ cells per well in a 96-well plate and treated for 3 hours with $0-10 \mu \mathrm{M}$ of
NS1 or gold nanostars ( $n=12$ each, where ' $n$ ' is a well of cells). Medium was replaced with fresh medium and cells were irradiated at a fluence of $1 \mathrm{~J} / \mathrm{cm}^{2}$. Following incubation in the dark for 18 hours, viability of the irradiated cells was assessed using the MTT assay as per manufacturer's instructions (Sigma Aldrich).

\section{Results and Discussion}

Since the energy difference between lattice structures of metal nanocrystals does not allow uniform growth structure during nucleation and growth process, a one-pot strategy was used to obtain 3-dimensional multibranched star-shaped gold nanocrystals 
(Nanostars). Such nanostars were synthesised to achieve longitudinal surface plasmon resonance (SPR) between 600-700 nm. Parameters including the concentration ratio of reducing agent to gold precursor $\left(\mathrm{R}_{[\mathrm{HEPES}] / \mathrm{HAuCl} 4]}\right), \mathrm{pH}$ and temperature were varied to analyse the nanostar formation. $\mathrm{R}_{[\mathrm{HEPES}] / \mathrm{HAuCl} 4]}$ was varied from 20-200, with the ratio of 200 exhibiting the desired multibranched star-shape and SPR wavelength (Figures $2 \mathrm{~A}$ and $2 \mathrm{~B}$ respectively). The absorption maximum gradually red shifted with increasing $\mathrm{pH}$ (up to $\mathrm{pH}$ 9) (data not shown). A pH of 7.4 was selected for further synthesis for two reasons; it is close to the physiological $\mathrm{pH}$ and, at this $\mathrm{pH}$, nanostars with narrower absorption bands and thus high monodispersity were produced. As shown in the TEM image in Figure 2A, the size of synthesised nanostars ranged from 30-45 $\mathrm{nm}$. While the number of branches ranged from 1-7, nanostars predominantly had 4 branches, as shown in the histogram as in Figure $2 \mathrm{C}$. The growth reaction of nanostars from nucleation to anisotropic formation, took approximately $60 \mathrm{~min}$. After 60 minutes of reaction time, the nanostars were recovered from reaction solution as further growth would lead to the loss of anisotropic configuration through Ostwald ripening. Recovered nanostars had a zeta potential of -40.0 $\mathrm{mV}$, suggestive of higher dispersion stability in solution [27].

Gold nanostars enhanced the Raman signal of the well-studied Raman reporter, DTTCI (3,3'-diethyltrithiacarbocyanine iodide) considerably. An increased SERS signal is evident in Figure 3A with increased DTTCI concentrations with constant nanostar concentrations. By comparison of the relative enhancement of four signature DTTCI Raman bands (marked arrows in Figure 3A), a $5 \mu \mathrm{M}$ concentration displayed optimum SERS signal (Figure 3B).

Stabilisation of nanostars prevents desorption of Raman reporter from its surface and solvent derived aggregation of the nanoconstruct. Various biopolymers have been reported as stabilisers, but polyethylene glycol (PEG) and bovine serum albumin (BSA) have been recently exploited for their enhanced biocompatibility $[9,28]$. PEG covering has been shown to stabilise gold nanoparticles and shield the Raman reporter due to the specific adherence on gold nanoparticle's surface that spontaneously forms a monolayer on gold [29]. BSA, in its denatured form, was found to be more advantageous in that its thiol binding capacity was found to be much stronger and maintained the non-aggregated isolated state of the nanostructures even after multiple washes. In the study by Yuan et al. nanoparticle tracking analyses (NTA) confirmed this and also showed that PEG stabilised nanoconstructs tend to form small clusters after multiple washes [9]. The stabilizing property of BSA stems from the exposed thiol groups. These can be attained by chemical denaturation of BSA which converts the multiple disulphide (S-S) bonds to functional thiol groups (-SH). These have been widely reported to have high affinity for the gold nanoparticle surface $[9,30]$. To prevent fluorescence quenching of photosensitisers (PS) by nanostars $[31,32]$ and to stabilise the nanoconstruct, dBSA was first conjugated with hypericin and the dBSA - hypericin complex was then conjugated with the DTTCI tagged nanostars. The concentration ratio of hypericin to be conjugated with dBSA was determined first. By keeping the dBSA concentration stable, various molar ratios (dBSA:hypericin) from 1:2 (higher concentration of hypericin) to 8:1 (lower concentration of hypericin) were examined. The concentration ratio of 4:1 was found to be optimal (Figure 4), as any further increase in hypericin concentration led to fluorescence self-quenching. This methodology of conjugating hypericin with dBSA to prevent fluorescence quenching by nanostars was corroborated by another group by [26] who investigated the conjugation site of hypericin within serum albumins and reported that hypericin binds with the deeply localized IIA sub-domain of BSA. This study also investigated SERS of hypericin when conjugated with BSA-hypericin complex. A strong reduction in intensity of hypericin's Raman bands when in complex with BSA was observed using SERS spectroscopy which is indicative of its greater distance from nanoparticle's surface resulting from its conjugation with BSA [26]. Thus, conjugating hypericin with BSA provided the dual purpose of stabilizing nanostars and also localizing hypericin in deep pockets within BSA, enabling hypericin to retain its fluorescence.

Quantification of Au (III) in nanostars and the NS was analysed using AAS and found to be $19 \mathrm{ppm}$ and $4 \mathrm{ppm}$ respectively. The lesser yield with NS was understandable as colloidal nanostars were washed multiple times after every step in fabrication of the NS and loss of some nanostars was unavoidable. Using UV-Vis standard curve analysis and the aforementioned quantification of gold in NS, the number of hypericin molecules conjugated with each nanostar was calculated to be 6250 .
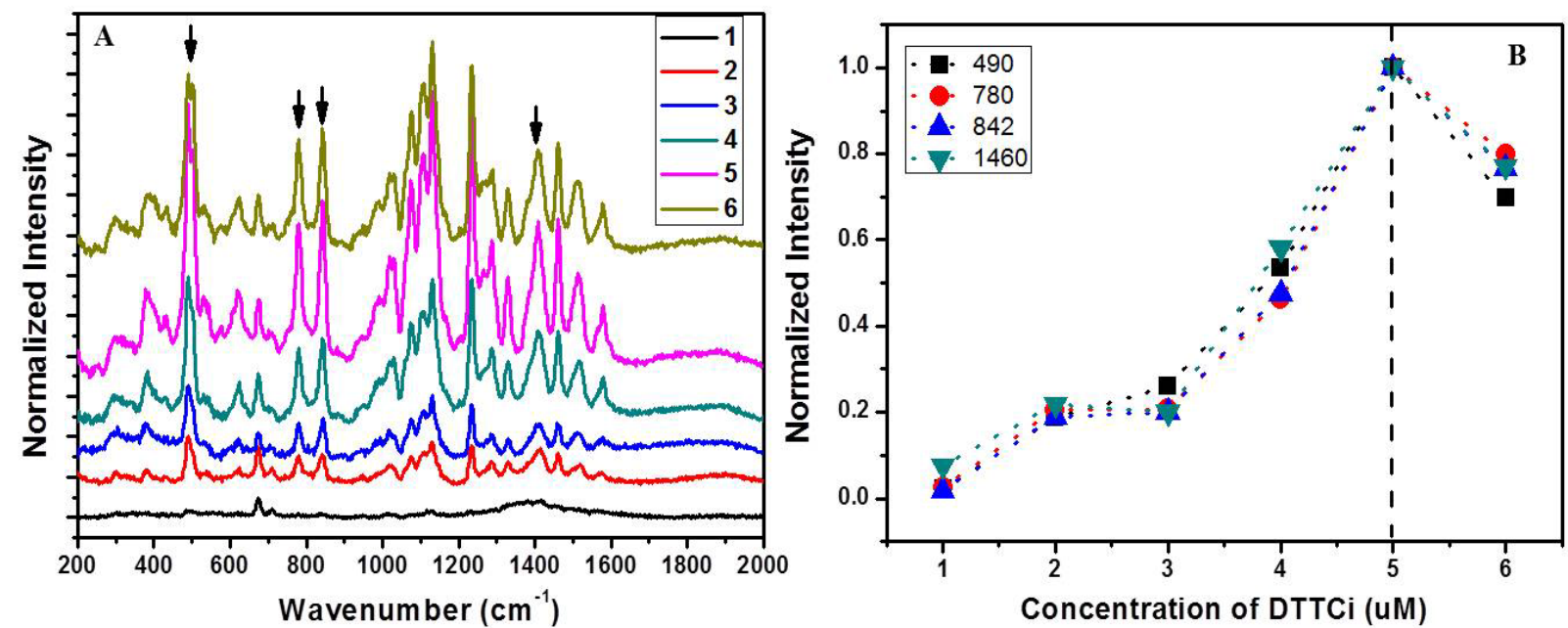

Figure 3: SERS analysis - Enhancement of SERS spectra at different concentrations of DTTCl by gold nanostars (A). Plot displaying intensity enhancement of 4 signature Raman bands of DTTCI by gold nanostars (B). 
Citation: Raghavan V, Connolly JM, Fan HM, Dockery P, Wheatley A, et al. (2014) Gold Nanosensitisers for Multimodal Optical Diagnostic Imaging and Therapy of Cancer. J Nanomed Nanotechnol 5: 238. doi: 10.4172/2157-7439.1000238
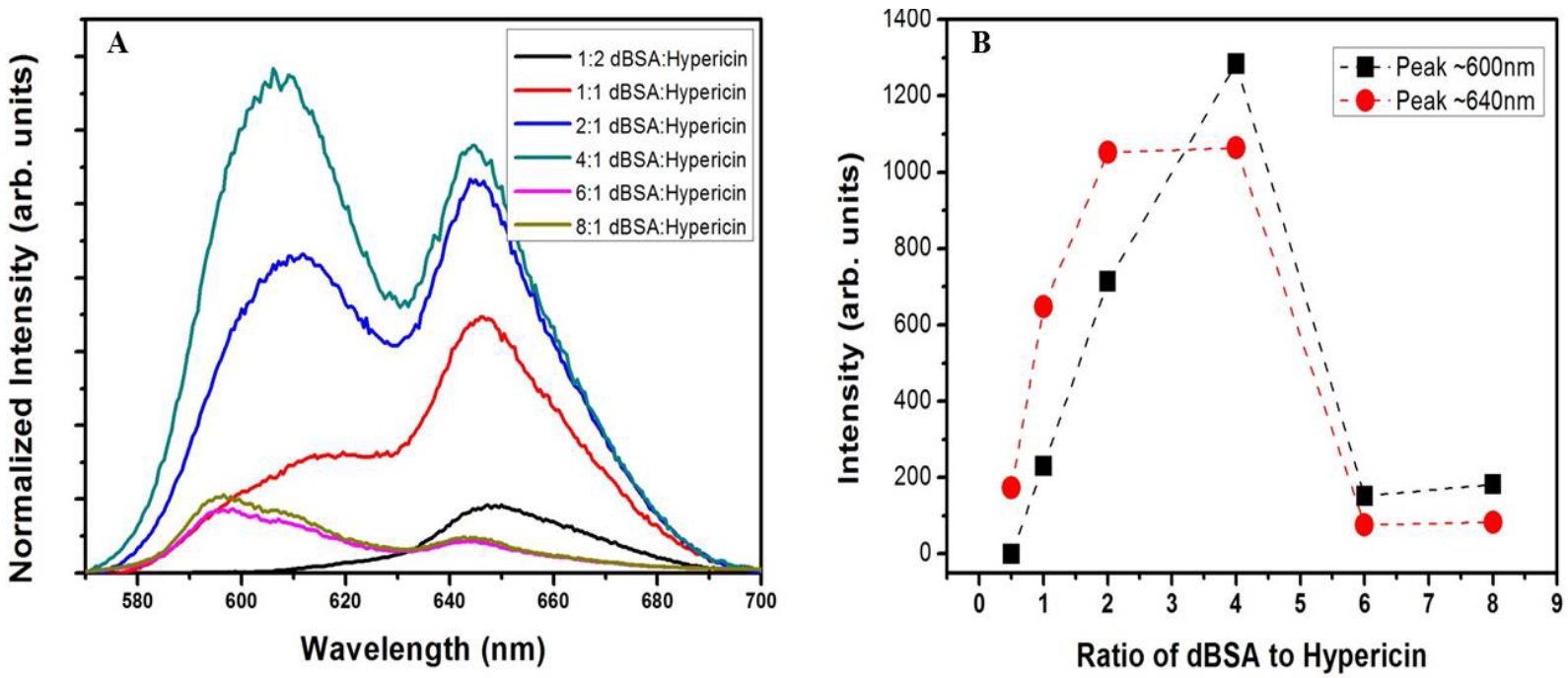

Figure 4: Hypericin conjugation with BSA - Absorption spectra of different concentration ratios of the dBSA:Hypericin complex (A). Scatter plot displaying maximum intensity of absorption bands with the concentration ratio of 4:1 (B).

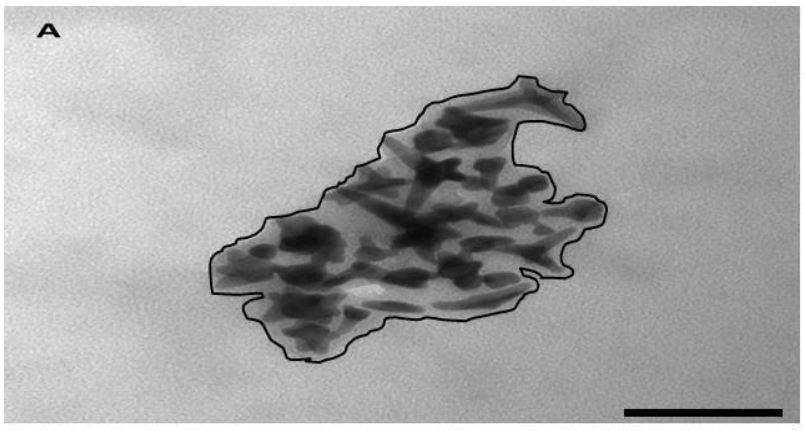

C

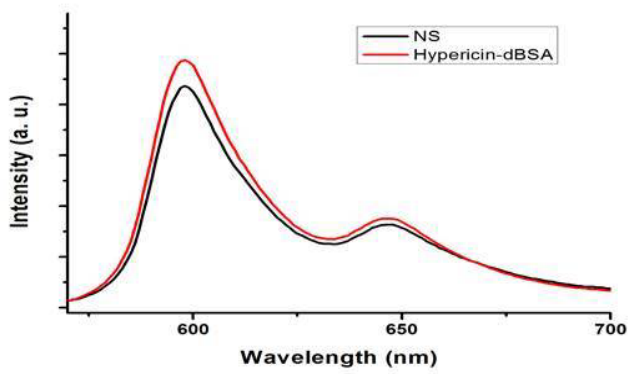

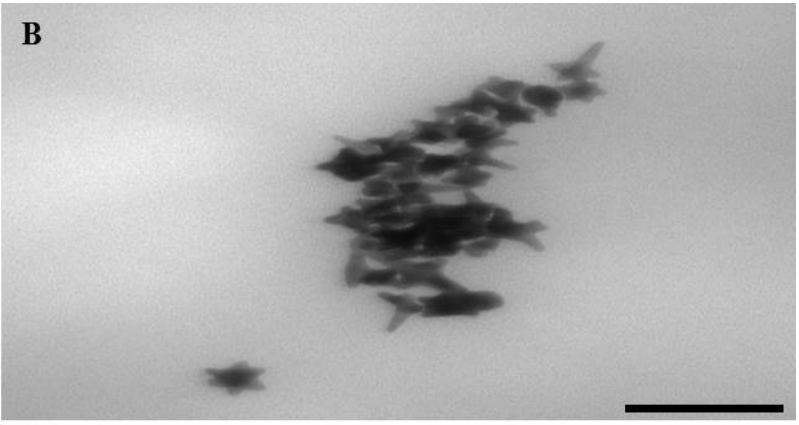

D

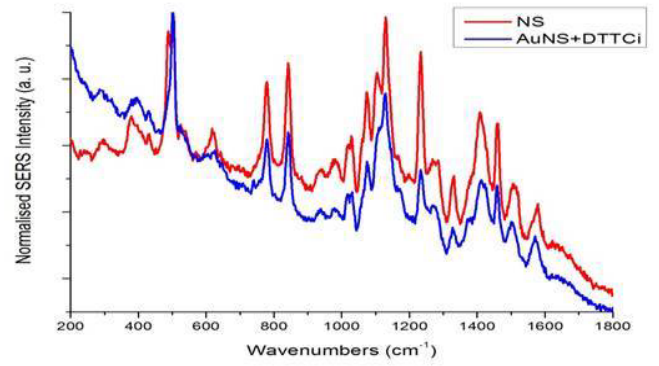

Figure 5: Nanosensitiser Characterisation - TEM image of NS (A) with the marking showing the negatively stained dBSA envelope (scale bar: 100 nm) and that of unstained control (B); Comparison of fluorescence spectra of hypericin-dBSA with NS (C). Retention of SERS enhancement of DTTCI by gold nanostars before and after fabrication of NS (D).

The NS were characterised for their structural stability and retention of optical properties of the individual components. TEM imaging of the NS clearly showed the stabilizing envelope of dBSA around nanostars. The nanosensitisers were negatively stained with $0.5 \%$ uranyl acetate, a standard negative stain for proteins, to observe the BSA coating around nanostars; unstained nanosensitiser sample was also imaged as a negative control (Figures $5 \mathrm{~A}$ and $5 \mathrm{~B}$ respectively). Fluorescence retention of hypericin after the fabrication of NS was examined, with the NS displaying similar spectra as hypericin conjugated with dBSA
(Figure 5C) with little decrease in intensity which might be due to the unbinding of hypericin molecules during purification process. SERS capability of nanostars before and after NS fabrication was examined. As can be seen in Figure 5D, the nanostar which is the core of the NS displayed similar SERS enhancement of DTTCI as did colloidal nanostars.

Singlet oxygen $\left({ }^{1} \mathrm{O}_{2}\right)$ generation capability and thus photodynamic efficacy of the NS in solution was investigated with laser irradiation 
Citation: Raghavan V, Connolly JM, Fan HM, Dockery P, Wheatley A, et al. (2014) Gold Nanosensitisers for Multimodal Optical Diagnostic Imaging and Therapy of Cancer. J Nanomed Nanotechnol 5: 238. doi: 10.4172/2157-7439.1000238

Page 6 of 8

at $543 \mathrm{~nm}\left(2.0 \mathrm{~W} / \mathrm{cm}^{2}\right)$, closely matching the absorption wavelength of hypericin. Singlet oxygen generation was demonstrated by the increase in fluorescence intensity of singlet oxygen sensor green (SOSG) at a $532 \mathrm{~nm}$ wavelength in the presence of singlet oxygen in solution [33]. Equimolar concentrations of pure hypericin with SOSG was investigated and showed a gradual increase of fluorescence signal up to $60 \mathrm{~min}$ (Figure 6A). A similar pattern was demonstrated with NS (Figure 6B) showing the efficacy of hypericin in terms of ${ }^{1} \mathrm{O}_{2}$ generation with increasing irradiation time when in pure solution and also post-fabrication of NS. Though the fluorescence signal of hypericin decreased after persistent irradiation (upto $60 \mathrm{~min}$ ) due to photobleaching (data not shown), the capability of ${ }^{1} \mathrm{O}_{2}$ generation stayed relatively high, thus demonstrating the NS's potential as a viable PDT agent. Unlike typical nanoconstructs used for drug delivery, NSs are not required to release the PS upon chemical or physical stimuli $[4,34]$ for it to be therapeutically effective. As can be seen from the SOSG analysis, hypericin within the NS construct is capable of PDT activity via generation of significant amount of ${ }^{1} \mathrm{O}_{2}$.

The photothermal capability of NS was next investigated. After irradiating with $675 \mathrm{~nm}$ laser at $2.0 \mathrm{~W} / \mathrm{cm}^{2}$, the temperature of the NS solution rose rapidly from the initial temperature of $20^{\circ} \mathrm{C}$ to around $50^{\circ} \mathrm{C}$ within 20 minutes and then plateaued (Figure 6C). Under the same experimental conditions, pure hypericin and dBSA samples were also irradiated, and negligible increases in temperature were shown, thus confirming that the sharp rise in temperature was purely through the photothermal effect of nanostars. With NS exerting combinatorial PDT and PTT capability, the therapeutic efficacy can be managed by adjusting the irradiation time.

In vitro studies were then performed to examine the uptake of NS and retention of hypericin's fluorescence upon uptake by the cells. Confocal fluorescence microscopy (CFM) images showed that NS with hypericin concentration of $5 \mu \mathrm{M}$ can be detected in cytoplasm of the cells and not inside the nucleus (Figure 7A). Similar uptake can be seen with the positive control of NS with same concentration of hypericin but enveloped with PEG instead of dBSA (Figure 7B), which shows that dBSA stabilisation does not affect the NS uptake and hypericin's fluorescence within the cells. The uptake of NS within cells and its distribution in the cytoplasm can also be examined using dark-field microscopy (DFM), which makes use of the back-scattering from the gold nanostars that form the core of the NS. As seen in CFM images,
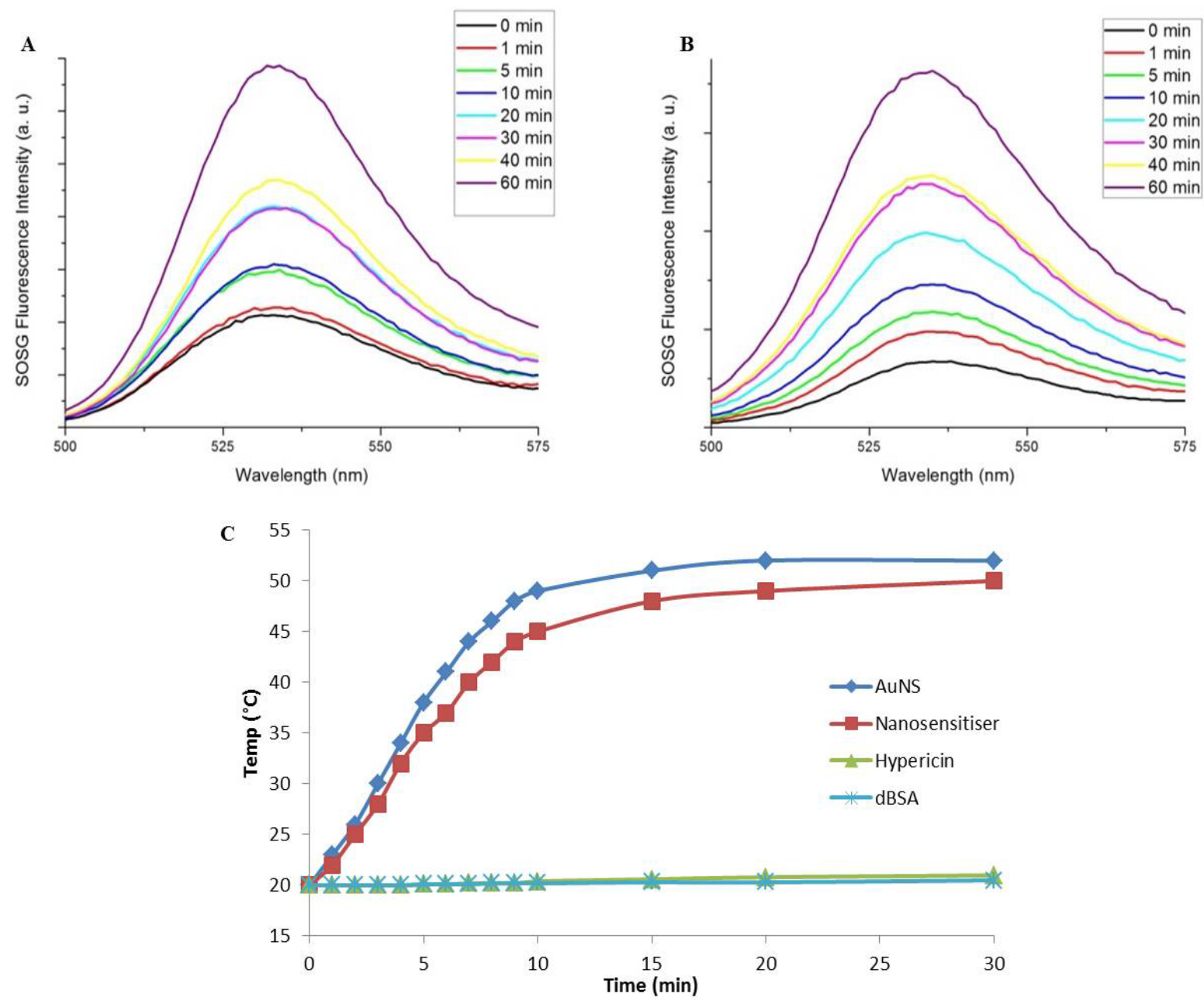

Figure 6: Photodynamic and photothermal capability of the NS - SOSG fluorescence spectra of pure hypericin (A) and NS (B) excited with 543 nm laser at different irradiation times $\left(2.0 \mathrm{~W} / \mathrm{cm}^{2}\right)$. Temperature curves of equal concentration of pure gold nanostars and gold nanostars in NS, pure hypericin, and denatured $\mathrm{BSA}$, all irradiated with $675 \mathrm{~nm}$ laser at $2.0 \mathrm{~W} / \mathrm{cm}^{2}(\mathrm{C})$. 
Citation: Raghavan V, Connolly JM, Fan HM, Dockery P, Wheatley A, et al. (2014) Gold Nanosensitisers for Multimodal Optical Diagnostic Imaging and Therapy of Cancer. J Nanomed Nanotechnol 5: 238. doi: 10.4172/2157-7439.1000238
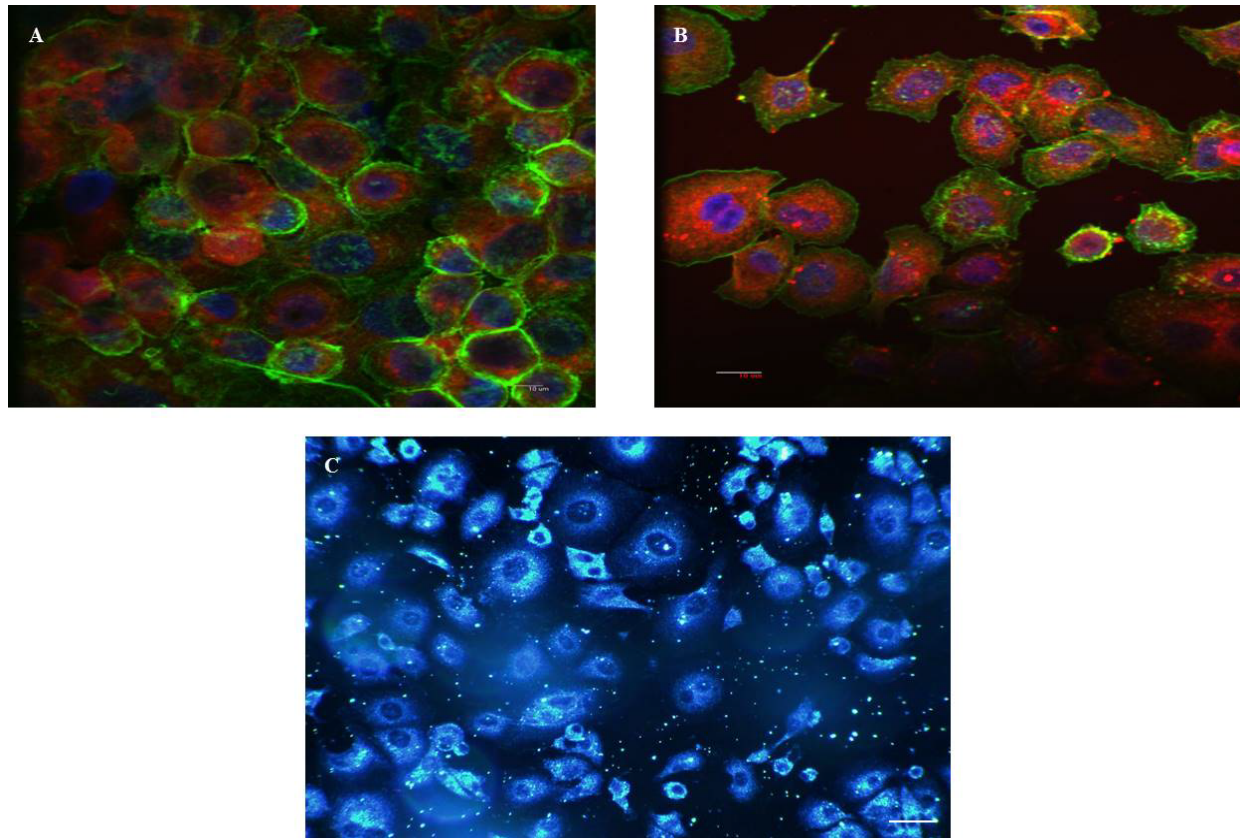

Figure 7: In vitro imaging of the NS - Confocal fluorescence microscopy images of NS with hypericin concentration of $5 \mu \mathrm{M}$ coated with dBSA (A), and of NS with hypericin concentration of $5 \mu \mathrm{M}$ coated with PEG (B). Green fluorescence from phalloidin green staining actin and blue fluorescence from nuclear stain Hoechst 33342. Scale bar $=1$ micron. Dark-field microscopy image of NS (C). Scale bar of 10 microns.
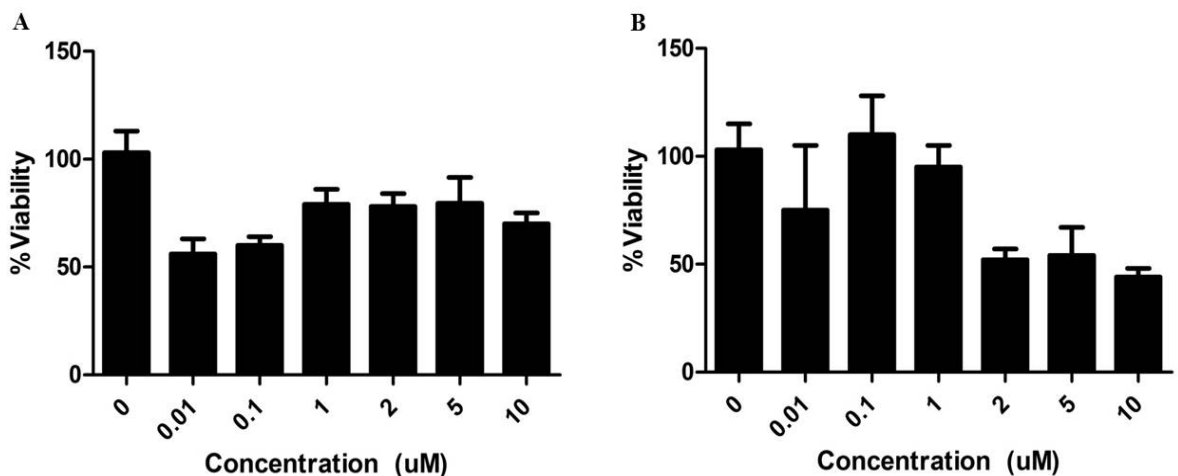

Figure 8: In vitro PDT and PTT - Percentage cell viability after PDT (A) and PTT (B) treatments with various concentrations $(0-10 \mu \mathrm{M})$ of hypericin in NS for PDT and gold nanostars in NS for PTT ( $n=12$ each).

scattering from nanostars in NS is evident in the cytoplasm of the cells and not within the nucleus (Figure $7 \mathrm{C}$ ). Figures $8 \mathrm{~A}$ and $8 \mathrm{~B}$ show the percentage cell viability compared to untreated control following PDT and PTT treatment with NS containing $0-10 \mu \mathrm{M}$ hypericin and gold nanostars, respectively. A significant PDT effect can be seen using as little as $0.01 \mu \mathrm{M}$ concentration of hypericin, and a significant PTT effect is evident using $2 \mu \mathrm{M}$ of gold nanostars. More detailed in vitro analyses including NS detection with confocal Raman microscopy (CFM) and TEM imaging, concentration dependant uptake of NS with CFM and DFM, and in vivo therapeutic efficiency are all in progress.

\section{Conclusion}

A novel methodology for the synthesis and fabrication of anisotropic gold nanostar based nanoprobes (NS) for multimodal detection and therapy for cancer has been developed. Multibranched gold nanostars were synthesised based on a green chemistry approach using HEPES as a reducing and shape directing agent. After coating with a monolayer of the NIR Raman dye DTTCI, nanostars significantly enhanced the SERS signal of DTTCI's signature Raman bands. Conjugation of hypericin to denatured BSA before being fabricated into the nanoconstruct offered the dual purpose of stabilising the structure and preventing the fluorescence quenching of the photosensitiser. Physio-chemical and optical properties of the individual components were also investigated post-fabrication, demonstrating retention of NS stability. Synthesised NSs displayed SERS capability by its gold nanostar core and fluorescence capability by the photosensitier and as such can be used in SERS microscopy and photodynamic diagnosis (PDD), respectively for the detection of cancer. NSs also displayed therapeutic potential through the photodynamic capability of photosensitisers and photothermal capability of the multibranched gold nanostars. This nanoconstruct 
Citation: Raghavan V, Connolly JM, Fan HM, Dockery P, Wheatley A, et al. (2014) Gold Nanosensitisers for Multimodal Optical Diagnostic Imaging and Therapy of Cancer. J Nanomed Nanotechnol 5: 238. doi: 10.4172/2157-7439.1000238

not only exhibits the aforementioned theranostics capabilities, but further modalities including optical coherence tomography (OCT) and photoacoustic imaging could also be exploited owing to the gold nanostar's property of high scattering cross-section providing enhanced optical contrast. Preliminary in vitro analyses shown here demonstrate a real promise for NS as single node multimodal theranostic tool. Although the concept of multimodal nanophotosensitisers as clinical tools is in its initial stages, the exploitation of innovative nanocarriers and methodologies for fabrication shows promise and may attract the attention of the clinical community.

\section{References}

1. Robertson CA, Evans DH, Abrahamse H (2009) Photodynamic therapy (PDT): a short review on cellular mechanisms and cancer research applications for PDT. J Photochem Photobiol B 96: 1-8.

2. Kubin A, Wierrani F, Burner U, Alth G, Grünberger W (2005) Hypericin--the facts about a controversial agent. Curr Pharm Des 11: 233-253.

3. Konan YN, Gurny R, Allémann E (2002) State of the art in the delivery of photosensitizers for photodynamic therapy. J Photochem Photobiol B 66: 89106.

4. Lim CK, Heo J, Shin S, Jeong K, Seo YH, et al. (2013) Nanophotosensitizers toward advanced photodynamic therapy of Cancer. Cancer Lett 334: 176-187.

5. Huang X, Jain PK, El-Sayed IH, El-Sayed MA (2007) Gold nanoparticles: interesting optical properties and recent applications in cancer diagnostics and therapy. Nanomedicine (Lond) 2: 681-693.

6. Sajanlal PR, Sreeprasad TS, Samal AK, Pradeep T (2011) Anisotropic nanomaterials: structure, growth, assembly, and functions. Nano Rev 2.

7. Jeong GH, Lee YW, Kim M, Han SW (2009) High-yield synthesis of multibranched gold nanoparticles and their surface-enhanced Raman scattering properties. J Colloid Interface Sci 329: 97-102.

8. Schütz M, Steinigeweg D, Salehi M, Kömpe K, Schlücker S (2011) Hydrophilically stabilized gold nanostars as SERS labels for tissue imaging of the tumor suppressor p63 by immuno-SERS microscopy. Chem Commun (Camb) 47: 4216-4218.

9. Yuan H, Liu Y, Fales AM, Li YL, Liu J, et al. (2013) Quantitative surfaceenhanced resonant Raman scattering multiplexing of biocompatible gold nanostars for in vitro and ex vivo detection. Anal Chem 85: 208-212.

10. Luo Z, Fu T, Chen K, Han H, Zou M (2011) Synthesis of multi-branched gold nanoparticles by reduction of tetrachloroauric acid with Tris base, and their application to SERS and cellular imaging. Microchimica Acta 175: 55-61.

11. Schlücker S (2009) SERS microscopy: nanoparticle probes and biomedical applications. Chemphyschem 10: 1344-1354.

12. Kho KW, Fu CY, Dinish US, Olivo M (2011) Clinical SERS: are we there yet? J Biophotonics 4: 667-684

13. Link S, El-Sayed MA (2000) Shape and size dependence of radiative, nonradiative and photothermal properties of gold nanocrystals. International Reviews in Physical Chemistry 19: 409-453.

14. Kah JCY, Wan RCY, Wong KY, Mhaisalkar S, Sheppard CJR, et al. (2008) Combinatorial treatment of photothermal therapy using gold nanoshells with conventional photodynamic therapy to improve treatment efficacy: an in vitro study. Lasers in surgery and medicine 40: 584-589.

15. Kuo WS, Chang YT, Cho KC, Chiu KC, Lien CH, et al. (2012) Gold nanomaterials conjugated with indocyanine green for dual-modality photodynamic and photothermal therapy. Biomaterials 33: 3270-3278.

16. Kim JY, Choi WI, Kim M, Tae G (2013) Tumor-targeting nanogel that can function independently for both photodynamic and photothermal therapy and its synergy from the procedure of PDT followed by PTT. J Control Release 171: 113-121.

17. Bhuvaneswari R, Gan YY-Y, Yee KKL, Soo KC, Olivo M (2007) Effect of hypericin-mediated photodynamic therapy on the expression of vascular endothelial growth factor in human nasopharyngeal carcinoma. International journal of molecular medicine 20: 421-428.

18. Kamuhabwa A, Agostinis P, Ahmed B, Landuyt W, Van Cleynenbreugel B, et al (2004) Hypericin as a potential phototherapeutic agent in superficial transitional cell carcinoma of the bladder. Photochemical \& Photobiological Sciences 3 : 772-780.
19. Karioti A, Bilia AR (2010) Hypericins as potential leads for new therapeutics. Int J Mol Sci 11: 562-594.

20. Usuda J, Kato H, Okunaka T, Furukawa K, Tsutsui H, et al. (2006) Photodynamic therapy (PDT) for lung cancers. J Thorac Oncol 1: 489-493.

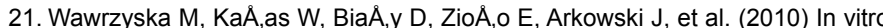
photodynamic therapy with chlorin e6 leads to apoptosis of human vascular smooth muscle cells. Arch Immunol Ther Exp (Warsz) 58: 67-75.

22. Sheleg SV, Zhavrid EA, Khodina TV, Kochubeev GA, Istomin YP, et al. (2004) Photodynamic therapy with chlorin e(6) for skin metastases of melanoma. Photodermatol Photoimmunol Photomed 20: 21-26.

23. Moon YH, Kwon SM, Kim HJ, Jung KY, Park JH, et al. (2009) Efficient preparation of highly pure chlorin e6 and its photodynamic anti-cancer activity in a rat tumor model. Oncol Rep 22: 1085-1091.

24. Xie J, Lee JY, Wang DI (2007) Seedless, surfactantless, high-yield synthesis of branched gold nanocrystals in HEPES buffer solution. Chemistry of materials 19: 2823-2830.

25. Gao X, Chan WC, Nie S (2002) Quantum-dot nanocrystals for ultrasensitive biological labeling and multicolor optical encoding. J Biomed Opt 7: 532-537.

26. Miskovsky P, Hritz J, Sanchez-Cortes S, Fabriciova G, Ulicny J, et al. (2001) Interaction of hypericin with serum albumins: surface-enhanced Raman spectroscopy, resonance Raman spectroscopy and molecular modeling study. Photochem Photobiol 74: 172-183.

27. Zhang Y, Yang M, Portney NG, Cui D, Budak G, et al. (2008) Zeta potential: a surface electrical characteristic to probe the interaction of nanoparticles with normal and cancer human breast epithelial cells. Biomed Microdevices 10 321-328.

28. Neng J, Harpster MH, Wilson WC, Johnson PA (2013) Surface-enhanced Raman scattering (SERS) detection of multiple viral antigens using magnetic capture of SERS-active nanoparticles. Biosens Bioelectron 41: 316-321.

29. Gandra N, Singamaneni S (2013) Bilayered Raman-intense gold nanostructures with hidden tags (BRIGHTs) for high-resolution bioimaging. Adv Mater 25: 1022-1027.

30. Xie J, Zhang Q, Lee JY, Wang DI (2008) The synthesis of SERS-active gold nanoflower tags for in vivo applications. ACS Nano 2: 2473-2480.

31. Dulkeith E, Morteani AC, Niedereichholz T, Klar TA, Feldmann J, et al. (2002) Fluorescence quenching of dye molecules near gold nanoparticles: radiative and nonradiative effects. Phys Rev Lett 89: 203002.

32. Schneider G, Decher G, Nerambourg N, Praho R, Werts MH, et al. (2006) Distance-dependent fluorescence quenching on gold nanoparticles ensheathed with layer-by-layer assembled polyelectrolytes. Nano Lett : 530-536.

33. Lin H, Shen Y, Chen D, Lin L, Wilson BC, et al. (2013) Feasibility study on quantitative measurements of singlet oxygen generation using singlet oxygen sensor green. J Fluoresc 23: 41-47.

34. Jang B, Park JY, Tung CH, Kim IH, Choi Y (2011) Gold nanorod-photosensitize complex for near-infrared fluorescence imaging and photodynamic/ photothermal therapy in vivo. ACS Nano 5: 1086-1094. 